BUHEP-98-28

MADPH-98-1075

\title{
Flavour Universal Dynamical Electroweak Symmetry Breaking
}

\author{
Gustavo Burdman* \\ Department of Physics, University of Wisconsin, Madison, WI 53706. \\ Nick Evans ${ }^{\dagger}$ \\ Department of Physics, Boston University, Boston, MA 02215.
}

\begin{abstract}
The top condensate see-saw mechanism of Dobrescu and Hill allows electroweak symmetry to be broken while deferring the problem of flavour to an electroweak singlet, massive sector. We provide an extended version of the singlet sector that naturally accommodates realistic masses for all the standard model fermions, which play an equal role in breaking electroweak symmetry. The models result in a relatively light composite Higgs sector with masses typically in the range of (400-700) GeV. In more complete models the dynamics will presumably be driven by a broken gauged family or flavour symmetry group. As an example of the higher scale dynamics a fully dynamical model of the quark sector with a GIM mechanism is presented, based on an earlier top condensation model of King using broken family gauge symmetry interactions (that model was itself based on a technicolour model of Georgi). The crucial extra ingredient is a reinterpretation of the condensates that form when several gauge groups become strong close to the same scale. A related technicolour model of Randall which naturally includes the leptons too may also be adapted to this scenario. We discuss the low energy constraints on the massive gauge bosons and scalars of these models as well as their phenomenology at the TeV scale.
\end{abstract}

*burdman@pheno.physics.wisc.edu

†nevans@budoe.bu.edu 


\section{Introduction}

The electroweak symmetry (EWS) of the standard model, which is a chiral symmetry of the fermions, is spontaneously broken by some as yet unknown mechanism. The introduction of an elementary Higgs scalar is technically unnatural. A natural explanation for the breaking of the chiral symmetry is a higher scale repeat of the dynamical breaking induced by QCD. This idea spawned technicolour models [1] of EWS breaking but these models ran into trouble since they typically introduce many extra electroweak doublet fermions whose presence is naively in conflict with precision experimental data from LEP and SLC [2]. With the discovery of the large top mass it was proposed that a top quark condensate [3], generated by some chiral symmetry breaking but not confining interaction (most likely a gauge group broken close to its critical scale for chiral symmetry breaking) might be responsible for EWS breaking. However, the value of the top quark mass is too small to generate the EWS breaking scale $v$. Introducing extra fermions in this fashion again leads to conflict with electroweak precision measurements. A further problem, also found in technicolour, is that attempts to generate the top-bottom mass splitting typically give rise to large custodial isospin violating effects in the massive sector [6]. Such effects are harshly constrained by the precision measurements of the $\rho$ parameter. A possible alternative is the combination of top condensation as the source of a large dynamical top quark mass, and technicolour as giving most of the EWS breaking. In this scenario, known as topcolor assisted technicolor [⿴囗⿰丿㇄口], the extended technicolour interactions give the top quark only a small fraction of its mass. On the other hand, it is still necessary to have a large number of techni-doublets in order to obtain the correct pattern of light quark masses. A variety of models of this type as well as their phenomenological consequences, have been studied in the literature [5].

Recently Dobrescu and Hill [7] have proposed a model in which a dynamically generated mass for the top quark does generate the full electroweak symmetry breaking scale but the correct top mass is obtained as a result of a see-saw mechanism with a heavy fermion sector. Perhaps of more significance, the model allows the origin of custodial symmetry breaking to be deferred to an EWS singlet sector where it cannot contaminate the $\rho$ parameter. In their model the top quark is treated in a unique fashion, its condensation being driven by a broken colour interaction unique to the third generation quarks. This essential nonuniversality disrupts the $S U(3)$ family symmetry of the standard model (SM) in the absence of fermion masses. As a consequence, it is not obvious how to feed the top condensate down to provide masses for the leptons and lighter first and second generation quarks.

In this paper we propose a mechanism analogous to the top condensate see-saw but which can naturally accommodate all the fermion masses and generation structure. An interesting aspect of the resulting models is that all the SM electroweak doublets play an equal role in 
breaking EWS. The result of this universal involvement in EWS breaking is that the models give rise to a relatively light巴 composite Higgs sector with a typical mass scale of order (400-700) GeV. The SM fermion mass splittings are the result of mass terms in an EWS singlet sector, which at this stage of model building is not explained dynamically but simply put in by hand. In Section 2 we present the simplest version of the model in which the dynamical symmetry breaking is driven by strong four fermion interactions. Depending on the flavour structure of the four fermion interactions driving EWS breaking, different patterns of pseudo-Nambu-Goldstone bosons (pNGB) result. We discuss in detail one possible pattern in Section 3 which is analogous to the spectrum of a one family technicolour model, and another one in the model of Section 4 where there are no un-eaten pNGB at the weak scale.

The simple model of Section 2 is in fact inspired by a more complete model of the dynamics of top condensation of King [8], which in turn was derived from a technicolour model by Georgi [9]. In Ref. [8] the four fermion interactions are the result of a broken gauged family symmetry. The biggest success of this class of models is that they have a GIM mechanism above the weak scale which protects them from flavour changing neutral currents (FCNC) even with family gauge bosons with masses of order a TeV. This is achieved by gauging the full chiral family symmetry, the symmetry responsible for the SM GIM mechanism. The model does have a number of possible flaws, including a non-trivial assumption about vacuum alignment and potentially light pNGB coming from the singlet sector. Nevertheless it is useful to elucidate the idea of a universal see-saw. The model is also suggestive of the gauge structure that is likely to underlie the universal see-saw model suggesting experimental searches. In Section 3 we revive the model by reinterpreting the dynamics when several gauge groups become strongly interacting at the same scale. The model then produces the flavour universal see-saw masses. The full model has additional interesting dynamics as a result of the massive, strongly interacting flavourons of the family gauge symmetry group and colourons analogous to those of the model of Ref. [14]. Their phenomenology, together with that of the scalar and pseudo-scalar sector originating from the breaking of the fermionic chiral symmetries, is discussed. The inclusion of leptons in that model is non-trivial. A related technicolour model of Randall [11], which more naturally includes the leptons, may be adapted to provide a low energy flavour universal EWS breaking model where the strong four fermion interactions result from a larger gauged flavour symmetry. We discuss this model, its flaws and its phenomenology in Section 4.

Finally, we must address the matter of fine tuning in top condensate models. In these models we will assume that the interactions responsible for EWS breaking are broken gauge

\footnotetext{
${ }^{1}$ We refer to Higgs masses in the few hundred GeV range as light, compared to the unitarity bound of approximately $\simeq 1.2 \mathrm{TeV}$. A large class of models of a strongly coupled EWS breaking sector saturate this bound.
} 
interactions with gauge bosons with masses in the range $\Lambda=(1-10) \mathrm{TeV}$. The fact that their interactions give rise to the weak scale $v=246 \mathrm{GeV}$ naively suggests fine tuning of order $(1-10) \%$. In fact in the underlying models we present, where the dynamics is more complete, a stronger degree of fine tuning is probably required both because gauge couplings do not run linearly with momentum scale and because several gauge groups become strongly coupled at essentially the same scale. Although the reader may still find these tunings uncomfortable they are clearly much less severe than those of the SM. We are agnostic and simply wish to explore this paradigm of model building. Thankfully in these matters we will eventually be instructed by experiment. One of the successes of the flavour universal models is that they allow direct condensation of all the electroweak doublets and hence masses for all the SM fermions without increasing the fine tuning. In the original top condensation models, to generate the electron mass by a direct condensate would have involved fine tuning of order $m_{e} / \Lambda$; the suppression of the mass in the present models is the result of the smallness of mass terms in a singlet sector. The structure also does not require very large singlet masses to see-saw the electron mass small which again would have been a source of fine tuning since it would have driven the upper cut-off higher.

\section{The Flavour Universal See-Saw Mechanism}

Our flavour universal see-saw model can be thought of as an extension of the top condensate see-saw model of Dobrescu and Hill [7], which we briefly review next. That model, in addition to the top doublet, $Q_{L}=\left(t_{L}, b_{L}\right)$ and the $t_{R}$ contains the electroweak singlets $\chi_{L}, \chi_{R}$ which have the same QCD and $U(1)_{Y}$ charges as the $t_{R}$. The EWS breaking is driven by the four fermion interaction

$$
G \bar{Q}_{L} \chi_{R} \bar{\chi}_{R} Q_{L}
$$

which, if the coupling $G$ is above critical, generates an EWS breaking mass between $Q_{L}$ and $\chi_{R}$. This can be seen in the large $N$ approximation to the gap equation

$$
\frac{1}{G}=\frac{N}{4 \pi^{2}}\left[\Lambda^{2}-m^{2} \ln \left(\frac{\Lambda^{2}}{m^{2}}\right)\right],
$$

where $\Lambda$ is the scale above which the dynamics generating Eq. (11) resides, and $m$ is the dynamically generated mass. In addition all EWS invariant masses are allowed, that is $\bar{t}_{R} \chi_{L}$ and $\bar{\chi}_{R} \chi_{L}$. The resulting mass matrix below the EWS breaking scale is then

$$
\left(\bar{t}_{L}, \bar{\chi}_{L}\right)\left(\begin{array}{cc}
0 & m_{t_{L} \chi} \\
m_{t_{R} \chi} & m_{\chi \chi}
\end{array}\right)\left(\begin{array}{c}
t_{R} \\
\chi_{R}
\end{array}\right)
$$


The value of $m_{t_{L} \chi}$ needed to provide all the EWS breaking vacuum expectation value (VEV) may be estimated using the Pagels-Stokar formula [15]

$$
v^{2} \simeq \frac{N_{c}}{4 \pi^{2}} m_{t_{L \chi}}^{2} \ln \left(\frac{\Lambda}{m_{\chi \chi}}\right) .
$$

Thus, for instance for $\Lambda / m_{\chi \chi} \simeq O(10), m_{t_{L} \chi} \approx 600 \mathrm{GeV}$. By appropriate choices of the remaining two masses (e.g. $m_{t_{R} \chi} \simeq 1 \mathrm{TeV}$ and $m_{\chi \chi} \simeq 3 \mathrm{TeV}$ ) the lightest mass eigenstate of the mass matrix comes out as $175 \mathrm{GeV}$, whereas the most massive is $3 \mathrm{TeV}$. In fact by suitable choices of the two singlet masses the value of the lightest eigenstate mass and the electroweak breaking mass may be maintained even in the limit of decoupling the $\chi$ fermion by taking $m_{\chi \chi} \rightarrow \infty$. Note that the electroweak breaking mass in that limit is only for the left handed top doublet so does not violate custodial isospin.

There is a crucial difference between the top see-saw model and previous top condensate models. That is that the EWS breaking VEV of the left handed top is with a EWS singlet not the right handed top. The right handed top also has a mass with a singlet sector fermion. The SM top mass results from the mass mixing in the singlet sector indirectly connecting the left and right handed tops. The size of the top mass is no longer a direct consequence of its role in EWS breaking but simply of some singlet mass structure. The top quark is therefore in this sort of model no longer the essential fermion to be involved in EWS breaking. Any SM fermion could play the same role! In the see-saw of Eq. (3) the light mass eigenstate is essentially given by $m_{t_{L} \chi} m_{t_{R} \chi} / m_{\chi \chi}$. Using the same see-saw but generating e.g. the electron mass would require a very large value of $m_{\chi \chi}$. The upper cut-off on the theory would have to be raised and with it the degree of fine tuning required to generate the weak scale. To avoid this we will enlarge the singlet sector. Consider for example the generation of the top quark mass. We introduce the additional electroweak singlet fields $\chi_{L}, \chi_{R}$ but also $\psi_{L}, \psi_{R}$. We assume these extra singlets are all coloured under the usual SU(3) QCD group and have the same hypercharge as the $t_{R}$. The model then induces four fermion interactions of the form (suppressing colour indices)

$$
\bar{Q}_{L} \chi_{R} \bar{\chi}_{R} Q_{L}
$$

In analogy with Eq. (1), these interactions become critical and break EWS. We also allow the singlet mass terms $\bar{t}_{R} \chi_{L}$ and similarly $\bar{\chi}_{L} \psi_{R}$ and $\bar{\chi}_{R} \psi_{L}$. Finally we include the mass term $M^{U} \bar{\psi}_{L} \psi_{R}$, which provides the only connection between the left and right handed top. Note that we have not included all possible singlet masses compatible with the gauge symmetries; effectively we have included one massive fermion $\left(\bar{\chi}_{L} \psi_{R}\right)$ that couples to the $t_{L}$ and another massive fermion $\left(\bar{\chi}_{R} \psi_{L}\right)$ that couples to the $t_{R}$. The two massive fermions then have small mass mixings from the $\bar{\psi}_{L} \psi_{R}$ masses which are the only couplings between these sectors. Although this may seem somewhat ad hoc at this stage the models of Sections 2 and 3 
naturally produce this mass structure. The important point here is that there is some singlet mass structure that can reproduce the SM fermion masses. The mass matrix takes the form

$$
\left(\bar{Q}_{L}, \bar{\chi}_{L}, \bar{\psi}_{L}\right)\left(\begin{array}{ccc}
0 & m_{1} & 0 \\
m_{2} & 0 & m_{3} \\
0 & m_{4} & M^{U}
\end{array}\right)\left(\begin{array}{c}
t_{R} \\
\chi_{R} \\
\psi_{R}
\end{array}\right)
$$

where $m_{1}$ is the EWS breaking mass. In general the matrix is complicated but the pattern of masses resulting can be seen by taking the decoupling limit. We imagine that the largest masses are $m_{3}$ and $m_{4}$ which bind the $\psi$ and $\chi$ fermions into two heavy Dirac fermions. We may then treat the remaining masses as perturbations. The SM top mass results from the diagram in Fig. 1 1 and the mass is proportional to the singlet mass $M^{U}$.

This singlet sector mass structure is more readily convertible to include the other SM fermions. We will write the SM quarks as $Q_{L}^{i}, U_{R}^{i}$ and $D_{R}^{i}$ where $i$ is a generation index. We may endow the fields $\chi$ and $\psi$ with a generation index and the mass matrices $m_{1}-m_{4}$ and $M^{U}$ with structure in the generation space. A four fermion operator of the form of Eq. (5) for each left handed doublet will break EWS through a condensate for each flavour. We shall assume that these operators give rise to flavour universal VEVs since we expect the four fermion operators to arise from some flavour universal broken gauge interaction. The precise way in which this dynamics will be implemented depends on the choice of broken gauge symmetry and we will discuss two possibilities: a gauged family symmetry and the gauging of the complete flavour symmetry. The dynamical implementation of these models and their consequences are discussed in detail in the next two sections.

The important point of the present structure is that the masses of the light eigenstates are proportional to the singlet mass matrix $M_{i j}^{U}$. Thus, the suppression of the up quark mass relative to the top quark mass only requires us to have a relatively smaller mass element in the $\psi$ mass matrix. The heaviest fermion masses $\left(m_{3}, m_{4}\right)$ do not contribute to the generation of the structure in the SM masses and hence the upper cut-off of the theory can be left unchanged even when we incorporate the lightest fermion masses. There is therefore no increase in the degree of fine tuning in the model if the up quark participates in EWS breaking. At least at this stage in the model building, the three generations of singlet fermions $\psi$ reflect the same mass hierarchy problem present in the spectrum of SM fermions. The important point is that the origin of the symmetry breaking mass splittings have been deferred to this EWS singlet sector. It is not our intention to address their origin here, though one could imagine generating these masses dynamically, via additional interactions residing at even higher energies, such as some singlet sector extended techincolour model or tumbling gauge structure. 


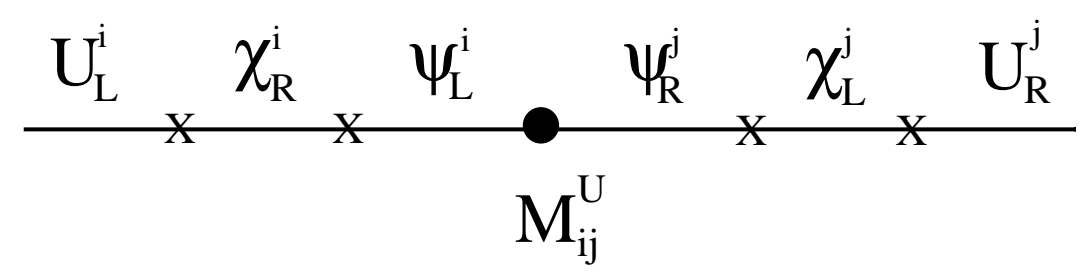

Figure 1: The mixing that produces the up quark sector masses.

Extending this scenario of mass generation to the isospin $-1 / 2$ quark sector is straightforward. We introduce the electroweak singlets $\omega_{L}^{i}, \omega_{R}^{i}$, as well as $\xi_{L}^{i}, \xi_{R}^{i}$, all with the same hypercharge assignments as right-handed down quarks. A four fermion interaction $\bar{Q}_{L}^{i} \omega_{R}^{i} \bar{\omega}_{R}^{j} Q_{L}^{j}$ will dynamically generate the condensate $\left\langle\bar{Q}_{L} \omega_{R}\right\rangle$ contributing to EWS breaking. The singlets $\xi$ have a mass matrix $M_{i j}^{D}$ which gives the connection between the left and right handed down-quark sectors. $M_{i j}^{D}$ generates the pattern of down quark masses through diagrams analogous to the one in Fig. 1 .

In this naive model with a four fermion interaction and explicit singlet masses, a CKM matrix may be easily incorporated. There are in total seven chiral family symmetries of the singlet fermions broken by six singlet mass matrices. These matrices can not therefore in general be diagonalized simultaneously and the model contains mixing angles that will feed into the SM sector.

The electroweak singlets, $\chi$ and $\psi$ in the up sector, and $\omega$ and $\xi$ in the down sector, have large masses relative to the weak scale (of order a few $\mathrm{TeV}$ ) and may be considered to be decoupled. The dynamical breaking of electroweak symmetry by the condensates between the SM left-handed fermions and the $\chi_{R}, \omega_{R}$ singlet fermions will generate a scalar sector of the low energy theory [3]. The precise form of the scalar sector is model dependent. We will discuss two examples of this sector in the following two sections where the origin of the dynamics is more concretely specified. However, it is possible to make a general point about the mass scale governing the masses of the scalar sector in relation to that of the top see-saw model. The important difference is that the EWS breaking VEV is flavour universal. In order to see this difference we notice that the Pagels-Stokar formula is given by

$$
v^{2}=\frac{N_{f}}{4 \pi^{2}} m_{1}^{2} \ln \left(\frac{\Lambda}{m_{3}}\right),
$$

where $N_{f}$ denotes the number of fermion flavours forming a condensate. For the topcondensate model, $N_{f}=N_{c}$ and we recover Eq. (4). On the other hand, for example in a model where all the quarks participate equally in EWS breaking $N_{f}=6 N_{c}$. For instance, for $\ln \Lambda / m_{3} \simeq \mathcal{O}(1)$ one obtains $m_{1} \simeq 360 \mathrm{GeV}$, whereas larger values of the ratio $\Lambda / m_{3}$ result in $m_{1} \simeq 250 \mathrm{GeV}$ or even lighter. This EWS breaking mass is approximately three 
times smaller than in the top see-saw model. If one naively computes the Higgs boson mass as in the large $N$ approximation to the four fermion theory the Higgs mass may be extracted from a bubble resummation and is found to be

$$
m_{h} \simeq 2 m_{1}
$$

implying Higgs masses in the range $m_{h} \simeq(500-700) \mathrm{GeV}$.

When the EWS breaking is driven by four fermion interactions it is easy to include the leptons $\left(L_{L}^{i}, N_{R}^{i}\right.$ and $\left.E_{R}^{i}\right)$ in the same pattern as the quarks. Additional EWS singlets are introduced analogous to $\chi^{i}, \psi^{i}, \omega^{i}$ and $\xi^{i}$ but with the SM interactions of the right handed leptons. Four fermion interactions analogous to those of (5) drive condensates involving $L_{L}^{i}$ and the massive singlet sector. The standard model lepton masses come again from the mass matrices linking the singlet $\psi$ fields. If the leptons participate equally in the EWS breaking condensates, then $N_{f}=6 N_{c}+6$ in the Pagels-Stokar formula, and the Higgs masses are controlled by a even lighter scale $\left(m_{h} \simeq 400-650 \mathrm{GeV}\right)$.

One of the benefits of this class of models is that there are only very small deviations from the SM values of electroweak precision variables such as the $S$ and $T$ parameters. The $S$ parameter essentially counts the number of electroweak doublets. The flavour universal model has only extra electroweak singlet fields so makes no extra contribution. By taking the singlet mass terms $\left(m_{3}, m_{4}\right)$ very large one may decouple them from the low energy dynamics (perhaps at the expense of increased fine tuning to generate $v$ ) or equivalently we may say that the physical heavy fermions have only a very small admixture of the electroweak doublets in them. In this limit the isospin breaking of the heavy fermions decouple from the $T$ parameter [7] leaving just the contribution from the light SM fermions with the standard masses. As this limit is relaxed all the mass eigenstates, the heavy and the light, will contribute to $T$ with by far the largest contribution from the top quark and its singlet partners. The calculation of $T$ is very similar to that of the top see-saw model and the additional contributions to $T$ are easily controlled to be of the order the experimental bounds for values of masses for the heaviest fermions of order $(1-5) \mathrm{TeV}$.

In the next two sections we present specific examples of the flavour universal see-saw mechanism, where the broken gauge dynamics is made explicit. In one case it results from a broken gauged family symmetry, in the other from a larger broken gauged flavour symmetry. A crucial aspect of theories with gauged flavour symmetries is to avoid the tight constraints on flavour changing neutral currents (FCNCs). We will provide models that achieve this by maintaining a GIM mechanism above the electroweak scale and provide existence proofs that such gauge dynamics is possible. We also discuss the phenomenology of these models, considering the consequences of the broken flavour symmetry as well as the scalar and pseudoscalar sector of the models. 


\section{Dynamics From Broken Family Symmetry}

Our first explicit example of a flavour universal EWS breaking model will assume that the family symmetry of the left handed SM fermions is gauged and broken at a scale of a few TeV. For simplicity we will restrict ourselves to the quark sector. The $\chi_{R}^{i}$ and $\omega_{R}^{i}$ fields are assumed to also transform under this gauge group. The broken gauged family interactions result in the four fermion interactions

$$
\bar{Q}_{L}^{i} \chi_{R}^{i} \bar{\chi}_{R}^{j} Q_{L}^{j}+\bar{Q}_{L}^{i} \omega_{R}^{i} \bar{\omega}_{R}^{j} Q_{L}^{j}
$$

where the family index is summed over. These interactions will generate the EWS breaking VEVs between the left handed quark multiplets and the singlet fermions.

The singlet sector masses are assumed to be in place to generate the quark mass matrices discussed in Section 2.

The scalar sector of the theory is expected to be large. The quark condensates are breaking an $S U(6)_{L} \times S U(6)_{R}$ chiral symmetry of the quarks and $\chi_{R}, \omega_{R}$ fields to the vector subgroup. To represent this as a Higgs model we must have a Higgs field transforming as a $(6, \overline{6})$ under the flavour group. There are thus 72 real scalars. As a result of the chiral symmetry breaking there are 35 NGB of which three are eaten to give masses to the $W$ and $Z$ gauge bosons. The remaining 32 pseudo-scalars are naively massless but acquire masses through SM gauge interactions. They correspond to a color-octet, $S U(2)_{L}$ singlet and a color-octet $S U(2)_{L}$ triplet. Their masses due to the strong interactions can be computed to be [16]

$$
m_{\pi}^{2}=3 \alpha_{s} M^{2}
$$

where $M$ is a high energy scale. For instance, for $M \simeq O(1) \mathrm{TeV}$, we obtain $m_{\pi} \simeq(500-$ $600) \mathrm{GeV}$. They couple to SM quarks with couplings proportional to $m_{q} / f_{\pi}\left(f_{\pi}\right.$ is the pNGB decay constant $\sim v$ ).

As discussed in Section 2 the non-NGB scalars are expected to have masses in the (500700) GeV range. One of these, a pseudo-scalar, is the would be NGB of the $U(1)_{A}$ symmetry, the remaining 36 scalars break down into the same SM gauge multiplets as the NGBs. We leave a more precise computation of the spectrum analogous to that of [3] for future investigation since it will be involved.

At the level of Eq. (9) the leptons may also be included in the interaction that breaks EWS. If the leptons participate in EWS breaking, then the flavour symmetry $S U(8) \times S U(8)$ is broken to the vector subgroup and there are 63 pNGB of which again only 3 are eaten. The additional 28 pNGB-s consist of: two electroweak triplets and two singlet leptoquarks 


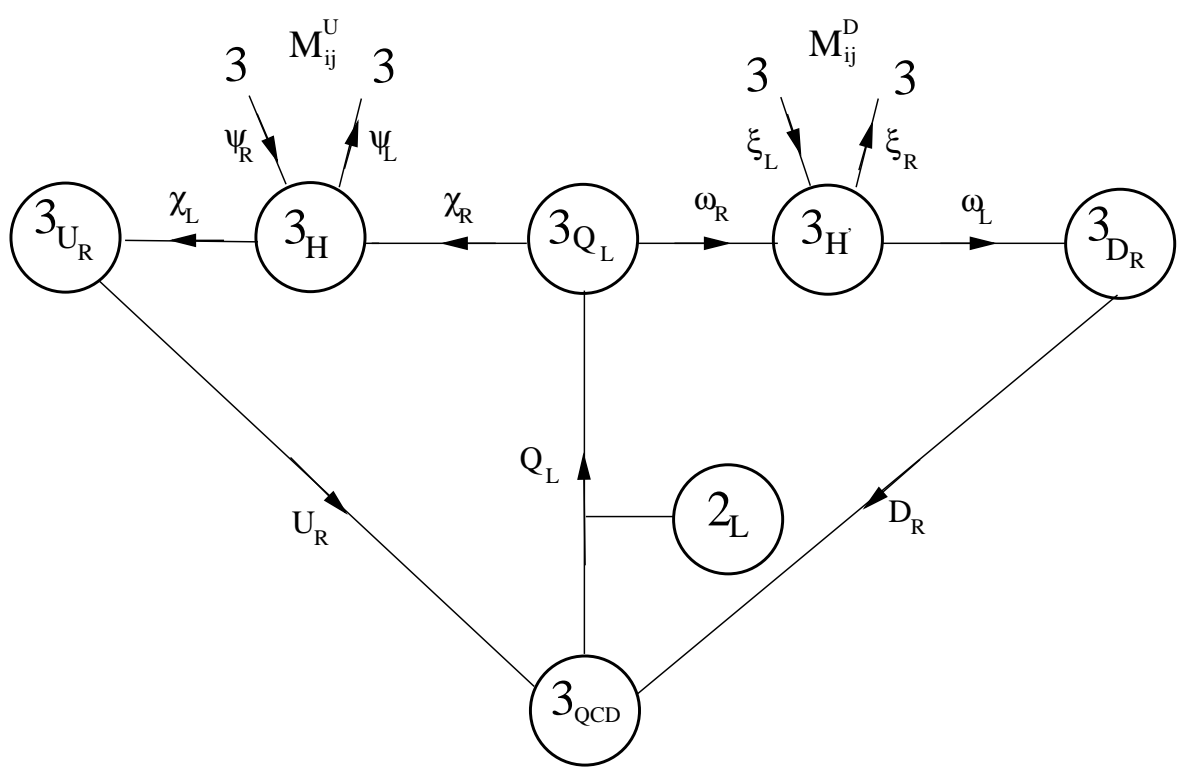

Figure 2: A dynamical model of the universal see-saw for the quark sector displayed in moose notation.

with masses

$$
m_{\pi}^{2}=\frac{4}{3} \alpha_{s} M^{2}
$$

that is $m_{\pi} \simeq(300-400) \mathrm{GeV}$; there is also a triplet and a singlet of colour neutral pNGB. These latter pNGB do not receive masses from the SM gauge interactions at one loop and are potentially in conflict with experiment. This may be an indication that the leptons do not participate in EWS breaking but instead receive their mass by some radiative mechanism from the quark sector. In addition in this case there would be 65 massive scalars.

\subsection{A Dynamical Model of the Universal See-saw}

In order to provide a more explicit, and renormalizable, model of the flavour universal seesaw mass pattern described in Section 2 we revive a model of top condensation by King (in turn based on the technicolour model of Georgi [9]). The model is shown in "moose" notation in Fig. 2. Recall that in moose notation a gauge group is represented by a circled number $(N$ for $S U(N)$ ) and left-handed fermions transforming in the (anti-) fundamental representation of that group as lines with (out-) inward pointing arrows. Global symmetry groups are un-circled numbers. For simplicity we only consider the quark sector of the theory until the end of this section.

In Ref. [8] the dynamics was assumed to occur in the following fashion. We concentrate on the isospin $+1 / 2$ sector for ease of discussion. The $S U(3)_{H}$ "hypercolour" gauge group 
under which $\chi$ and $\psi$ transform were assumed to get strong generating the condensates $\left\langle\bar{\chi}_{L} \psi_{R}\right\rangle$ and $\left\langle\bar{\chi}_{R} \psi_{L}\right\rangle$ and breaking the two chiral family symmetry $S U(3)$ groups to global groups. The formation of these condensates is not the preferred breaking pattern when the family symmetry groups are weakly coupled. However, there is some evidence that the effective potential favours this pattern when the family symmetry groups are strongly coupled at the symmetry breaking scale. This hypothesis was first put forward by Georgi [9], elaborated on in Ref. [10 and disputed in Ref. [13]. We shall assume that this pattern of condensation is correct. In the model of King these condensates were the only ones to form at this scale and served the purpose of breaking the family gauge groups leaving strongly coupled "flavourons". The flavourons of the two chiral family groups mixed through the $\bar{\psi}_{L} \psi_{R}$ mass matrix and the result was four fermion interactions between the isospin $+1 / 2$ quark sector with the strongest channel proportional to the largest eigenvalue of the $\psi$ mass matrix. This strongest interaction was assumed to generate a top quark condensate $\left\langle\bar{t}_{L} t_{R}\right\rangle$.

We now propose an alternative possibility. Since the family symmetry groups are strongly interacting and close to their chiral symmetry breaking scale when the hypercolour group breaks its chiral symmetries, it is not clear that the $\chi$ and $\psi$ fields should be integrated out of the dynamics of the family gauge groups. It is very plausible that the condensates $\left\langle\bar{U}_{R}^{i} \chi_{L}\right\rangle$ and $\left\langle\bar{U}_{L}^{i} \chi_{R}^{i}\right\rangle$ form as well. If we assume this, then the strong dynamics immediately produces the pattern of masses needed for the flavour universal see-saw model. Precisely how large the three different condensates are is a sensitive matter of the three gauge groups interrelated strong interactions. We shall assume that by appropriate choices of couplings at the hypercolour group's strong scale the desired values can be obtained. There is of course, as in all top condensate models envisaged to date, a serious problem of fine tuning in the assumption that many gauge groups become strongly interacting at almost an identical scale. We offer no excuses (though it might be more natural if the gauge dynamics are walking in nature) but put forwards this model as a simple existence proof of dynamics that might give rise to the required effective theory.

The existence of these additional condensates also breaks the $S U(3)$ colour group and the hypercolour group to their vector subgroup that then plays the role of the low energy QCD group. There are thus flavour universal colourons analogous to those in the model of Ref. [14. The NGB associated with the condensate $\left\langle\bar{U}_{R}^{i} \chi_{L}^{i}\right\rangle$ are eaten in the breaking of these two groups to the vector subgroup. The NGB from the condensate $\left\langle\bar{U}_{L}^{i}\left(\chi_{R}^{i}+\omega_{R}^{i}\right)\right\rangle$ are those already discussed above. Finally there are the 36 NGB associated with the $\langle\chi \psi\rangle$ condensates of which 16 are eaten to give masses to the two sets of family symmetry group gauge bosons. The remaining NGBs will acquire masses from the explicit mass matrix $M_{i j}^{U}$ and the family gauge interactions that explicitly break the chiral symmetry group. However, the mass matrix is off diagonal in the basis of the condensate and the gauge interactions 
chiral, so these symmetry breakings will only contribute to the pNGB masses at second order. These EWS singlet pNGBs may therefore only have masses of the order of a few GeV. We will live with their existence since they only occur in the singlet sector of this model, which is in any case only intended to illustrate the concept of a flavour universal seesaw. We do not believe they are necessarily a robust signature of this class of models.

The bottom quark sector of the model behaves similarly to the top quark sector and the relative mass differences in the final spectrum are trivially the result of different mass matrices included by hand for the $\psi$ and $\xi$ singlet fermions of the two sectors. Including mixing angles is non-trivial [9]. They will result if the singlet fermion mass matrices in the up and down sectors, $M_{i j}^{U}$ and $M_{i j}^{D}$ are not simultaneously diagonal. As shown in Fig. 2 the model has sufficient global symmetry to simultaneously diagonalize both mass matrices and there will be no mixing angles. An attempt to remedy this problem by including yet higher energy scale dynamics can be found in Ref. [9]. We wish to defer questions of such high scale dynamics and simply note that there does not appear to be any problem, in principle, preventing the generation of mixing angles from the mass matrices of the singlet sector.

The model has flavour symmetries gauged at a relatively low scale, $\mathcal{O}(1) \mathrm{TeV}$, which might naively be expected to generate FCNCs. However, as promoted in Ref. [9], the model has the same chiral flavour symmetries as the SM even above the weak scale (that is $[S U(3) \times U(1)]^{3}$ ) and hence a GIM mechanism that allows the neutral currents to be written in diagonal form. FCNC effects will be generated with the inclusion of the generic mass matrices $M^{U / D} i j$ but the leading effects are through the quark masses themselves, and therefore simply correspond to the SM contributions. The largest additional contributions are from mass mixings of the left and right family gauge groups that lead to four fermion operators between the up (down)type quarks mixing through $M^{D}\left(M^{U}\right)$ which rotations on those fields cannot diagonalize. The leading such term contributing to $\Delta S=2$ processes is for example

$$
\frac{1}{\Lambda_{H}^{6}} \bar{Q}_{L}^{i}\left(M^{U} M^{U \dagger}\right)_{i j} Q_{L}^{j} \bar{Q}_{L}^{k}\left(M^{U} M^{U \dagger}\right)_{k l} Q_{L}^{l}
$$

In addition to the suppression by the gauge boson mass these contributions are suppressed by a factor of the SM mass mixing matrix element over $50 \mathrm{GeV}$ to the fourth power. They are therefore much smaller than the SM contributions.

The inclusion of leptons in the model is not so straightforward. Naively one would simply repeat the model of the quark sector with the SM leptons transforming under the same family symmetry groups. However, this would introduce unacceptably large lepton flavour violating effects (e.g. $K^{0} \rightarrow e \mu$ ) since two multiplets would now transform under a single family symmetry group only broken at the few $\mathrm{TeV}$ scale. As an alternative, one could simply replicate the whole moose model for the lepton sector. Additional $\chi$ and $\omega$ 
fields transforming under their own hypercolour groups would need to be added and the lepton mass matrices would come out proportional to the mass matrix put in between the new $\omega$ fields. The only problem is that to cancel anomalies the hypercolour group must be a $U(1)$ group which is not asymptotically free. Rather than make the model more baroque to force the leptons in, we will leave the model at this point since the similar model of the next section succeeds more naturally in including the leptons, although not without similar problems.

\subsection{Phenomenological Considerations}

In this section we discuss the phenomenological constraints on the family symmetry generated flavour universal see-saw model as well as its potential signatures at future experiments. Here we concentrate on the model giving quark masses and EWS breaking, leaving the discussion of lepton masses for the next section. The model described in the previous section contains several new states. There are the fermion singlets, $\chi_{L, R}$ and $\psi_{L, R}$ connected to the up-quark sector, and $\omega_{L, R}$ and $\xi_{L, R}$ to the down-quark sector. As mentioned in Section 3 , these acquire large masses as a result of the condensates formed among them, induced by the strong up and down hypercolour groups. This, together with the fact that they are electroweak singlets, implies that they are not relevant to the low energy phenomenology. They are, at this stage, simply conduits to communicate between the left and right-handed quark sectors and to mix with the left-handed quark doublets and generate EWS breaking at the right scale.

\subsubsection{Flavourons}

The breaking of the three gauged family groups $S U(3)_{L}, S U(3)_{U_{R}}$ and $S U(3)_{D_{R}}$ by the formation of the necessary singlet fermion condensates, implies the existence of three new sets of massive gauge bosons. These are family octets coupling separately to left-handed quark doublets, right-handed up and right-handed down quarks. The masses of these "flavourons" are expected to be similar and are determined by the scale where the hypercolour groups break the family symmetries.

The couplings of the flavourons to the SM fermions are given by

$$
\mathcal{L}_{f}=-g_{L} L_{\mu} \overline{Q_{L}^{i}} \gamma^{\mu} t_{i j}^{A} Q_{L}^{j}-g_{U} R_{\mu}^{U} \overline{U_{R}^{i}} \gamma^{\mu} r_{i j}^{A} U_{R}^{j}-g_{D} R_{\mu}^{D} \overline{D_{R}^{i}} \gamma^{\mu} s_{i j}^{A} D_{R}^{j}
$$

where $L_{\mu}, R_{\mu}^{U}$ and $R_{\mu}^{D}$ are the flavourons corresponding to the gauge family groups $S U(3)_{L}$, $S U(3)_{U_{R}}$ and $S U(3)_{D_{R}}$ respectively; and $t^{A}, r^{A}$ and $s^{A}$ are their respective generators, with 
$A=1, . ., 8$. They simply are $t_{i j}^{A}=r_{i j}^{A}=s_{i j}^{A}=\lambda_{i j}^{A} / 2$, with $\lambda^{A}$ the generators of SU(3). The fact that flavourons are flavour-octet gauge bosons seems to indicate the existence of non-diagonal vertices. However these do not result in FCNC processes due to the presence of the remnant global $S U(3)$ family symmetries after the breaking of the gauged ones. These approximate global symmetries allow us to implement the GIM mechanism along the lines of the models of Ref. [9] and are only broken by fermion mass terms.

The flavourons acquire masses at the scale $\Lambda_{f}$ where the hypercolour groups break the family symmetry. Thus, they are expected to be in the TeV range. On the other hand, their couplings to fermions must be strong enough to generate the $\left\langle\bar{Q}_{L} \chi_{R}\right\rangle$ and $\left\langle\bar{Q}_{L} \omega_{R}\right\rangle$ condensates that break the EWS, as well as the $\left\langle\bar{U}_{R} \chi_{L}\right\rangle$ and $\left\langle\bar{D}_{R} \omega_{L}\right\rangle$ necessary to obtain the SM quark masses. We compute the criticality condition in the NJL approximation. Defining $\kappa_{a} \equiv g_{a}^{2} / 4 \pi$, with $a=L, U, R$, the couplings must satisfy

$$
\kappa_{a} \geq 2 \pi\left(\frac{N_{g}}{N_{g}^{2}-1}\right),
$$

where $N_{g}$ refers to the number of generations. The condition Eq. (14) has important phenomenological consequences. For instance, it gives a lower bound for the production cross section, for a fixed flavouron mass. At the same time it implies that the flavourons' widths are rather large. For instance the flavourons coupled to the left-handed quarks have a width given by

$$
\Gamma_{f} \simeq \frac{\kappa_{L}}{6} M_{F},
$$

which implies that the minimum width, given by the critical value of $\kappa_{L}$, is approximately $40 \%$ of its mass making it difficult for them to be detected as clear mass bumps in $p \bar{p}$ collisions at the Tevatron. The situation is somewhat better for the flavourons coupled to the righthanded up and down quarks. Their minimum widths are about half that of Eq. (15)). On the other hand and for all cases, the large couplings in Eq. (14) ensure large excesses in hadronic production of all flavours. The fact that flavourons are chirally coupled may produce a very distinct signal in high transverse momentum jets. At energies below the flavouron mass these excesses mimic the behavior of contact terms with specific chirality. The limits from the Tevatron Run I data as well as the reach of Run II is currently under study.

The presence of strongly coupled gauge bosons may be constrained by their contributions to the $T$ parameter. As pointed out in Ref. [6], this is so even when the interaction itself is isospin conserving. The exchange of the new gauge bosons gives a two loop radiative correction to the isospin violating contributions to the $\rho$ parameter coming from one-loop diagrams involving the SM fermions. We first notice that these types of contributions to $T$ are induced only by the gauge bosons that couple to left-handed fermions. Thus, only one of the three flavourons must be considered. The relevant contribution to the $T$ parameter is 
given by

$$
T=\frac{4 \pi}{s^{2} \theta_{W} c^{2} \theta_{W} M_{Z}^{2}}\left(\frac{\Pi_{L L}\left(m_{t}, m_{b}\right)}{2}-\frac{\Pi_{L L}\left(m_{t}, m_{t}\right)}{4}\right),
$$

where $\Pi_{L L}\left(m_{t}, m_{b}\right)$ and $\Pi_{L L}\left(m_{t}, m_{b}\right)$ refer to the left-handed vacuum polarizations involving one top and one bottom quark, and two top quarks in the loop respectively, and they are evaluated at zero momentum transfer. Following [6] we will approximate the calculation of the two-loop diagram by a product of two one loop diagrams obtained after shrinking the flavouron propagator. Then, the vacuum polarizations are

$$
\Pi\left(m_{t}, m_{b}\right)=\frac{\Pi\left(m_{t}, m_{t}\right)}{4} \simeq-\frac{1}{16 \pi^{3}} m_{t}^{4}\left(\log \frac{\Lambda_{f}}{m_{t}}\right)^{2} \frac{\kappa_{L}}{M_{F}^{2}},
$$

where the vacuum polarizations were approximated by their divergent behavior given by the leading logarithm. This results in

$$
T=\frac{m_{t}^{4}}{s^{2} \theta_{W} c^{2} \theta_{W} M_{Z}^{2}} \frac{1}{8 \pi^{2}}\left(\log \frac{\Lambda_{f}}{m_{t}}\right)^{2} \frac{\kappa_{L}}{M_{F}^{2}} .
$$

This constraint is compatible with $\Lambda_{f}$ being of the order of the $\mathrm{TeV}$ scale. For instance, if we take $\log \left(\Lambda_{f} / m_{3}\right) \simeq 1$, and we assume $m_{3} \simeq 1 \mathrm{TeV}$, then the induced $T$ parameter is

$$
T \simeq 0.06 \frac{\kappa_{L}}{M_{F}^{2}}
$$

The current determination of $T$ from electroweak precision measurements gives [17 $T=$ $(-0.11 \pm 0.16)$, assuming a $300 \mathrm{GeV}$ Higgs mass. Thus, we see from Eq. (14) and Eq. (19) that flavouron masses in the $1 \mathrm{TeV}$ range and above are not in contradiction with the electroweak precision data.

\subsubsection{Colourons}

The flavourons induce four-fermion interactions between quarks and singlet fermions which are supercritical and result in EWS breaking as well as in the breaking of the hypercolour groups in such a way that $S U(3)_{H} \times S U(3)_{H^{\prime}} \times S U(3)_{c}$ breaks down to the ordinary QCD interactions. This breaking pattern leaves two sets of color-octet massive gauge bosons, the "colourons", each of them coupling separately to the up and down quarks.

The two sets of colourons in this model present some similarities with the massive coloroctet gauge boson in the model of Ref. [14]. As with the flavourons, they will give contributions to the renormalization of SM $\rho$ parameter. The resulting bounds are similar to those discussed for the flavourons and are in the TeV range 2 Moreover, and similarly to the case

\footnotetext{
${ }^{2}$ The essential difference between the two cases resides in the fact that the same colourons couple to left and right handed fermions. As a result, the effect in Eq. (19) is larger by a factor of 3 for colourons.
} 
of the flavourons discussed above, the colouron couplings must be supercritical in order for the hypercolour groups to generate mass terms in the singlet sector. This constraint, here once again, will imply large production cross sections but also large widths. The width of either of the color octets is given by $\kappa_{C} M_{C}$, with $\kappa_{C}$, the coupling of the corresponding colouron, reflecting the embedding of $Q C D$ in the hypercolour group and above the critical value. The main difference with the flavouron case is that colourons interfere with the QCD gluon-mediated processes. The consequences of this interference at hadron colliders were first studied in Ref. [18] in the context of Topcolor models, and later investigated in Ref. [14] for the case of flavour universal color-octet gauge bosons. The phenomenology of the colouron sector of the model is very similar to the one described in this latter work.

\subsubsection{Scalars and pNGB}

Finally, in the model of Fig. 2 the breaking of the $S U(6)_{L} \times S U(6)_{R}$ chiral symmetry under which $Q_{L}, \chi_{R}$ and $\omega_{R}$ transform, by a four fermion interaction implies the existence of a $(6, \overline{6})$ Higgs boson. The 72 real degrees of freedom split into 3 NGBs eaten by the $W$ and $Z$ bosons, 32 color -octet pNGB with masses estimated above to be in the few hundred GeV range, one pNGB acquiring a large mass due to the $U(1)$ anomaly, and 36 scalars in the Higgs sector with masses estimated to lie between $(500-700) \mathrm{GeV}$.

These states couple to the SM fermions through Yukawa interactions of order $m_{f} / f_{\pi}$. In fact they are precisely the lightest states found in a one family technicolour model without techni-leptons, and are subject to the similar low energy constraints. The most significant bounds on this scalar sector come from the measurements of $\Gamma(Z \rightarrow b \bar{b})$ and the $b \rightarrow$ $s \gamma$ branching ratio. The dominant contributions correspond to the color-octet scalars and pseudo-scalars. For instance, the effect of considering one of these color-octets in the $b \rightarrow s \gamma$ transition, when combined with the $3 \sigma$ interval from the most recent CLEO measurement of the inclusive rate [19], $\operatorname{Br}(b \rightarrow s \gamma)=(3.15 \pm 0.35 \pm 0.32 \pm 0.26) 10^{-4}$, translates into a

lower bound of $440 \mathrm{GeV}$ on the mass of the charged states. The effects of adding all the scalar and pseudo-scalar states may tighten the bounds, depending on the scalar masses. However, large cancellations are also possible among the various contributions and the SM $b \rightarrow s \gamma$ amplitude. The additional information from $b \rightarrow s \ell^{+} \ell^{-}$decays, to be available in the near future from the next generation of $B$ physics experiments, will elucidate this possibility. On the other hand, the $R_{b}$ bounds are not affected by the these cancellations. For instance, the $3 \sigma$ constraint on the color-octet states (assuming equal masses for scalars and pseudo-scalars) translates into a mass limit of $700 \mathrm{GeV}$. However, if the central value of $R_{b}$ is taken to be the SM one, the mass constraint is considerably smaller, although still a few hundred $\mathrm{GeV}$. 


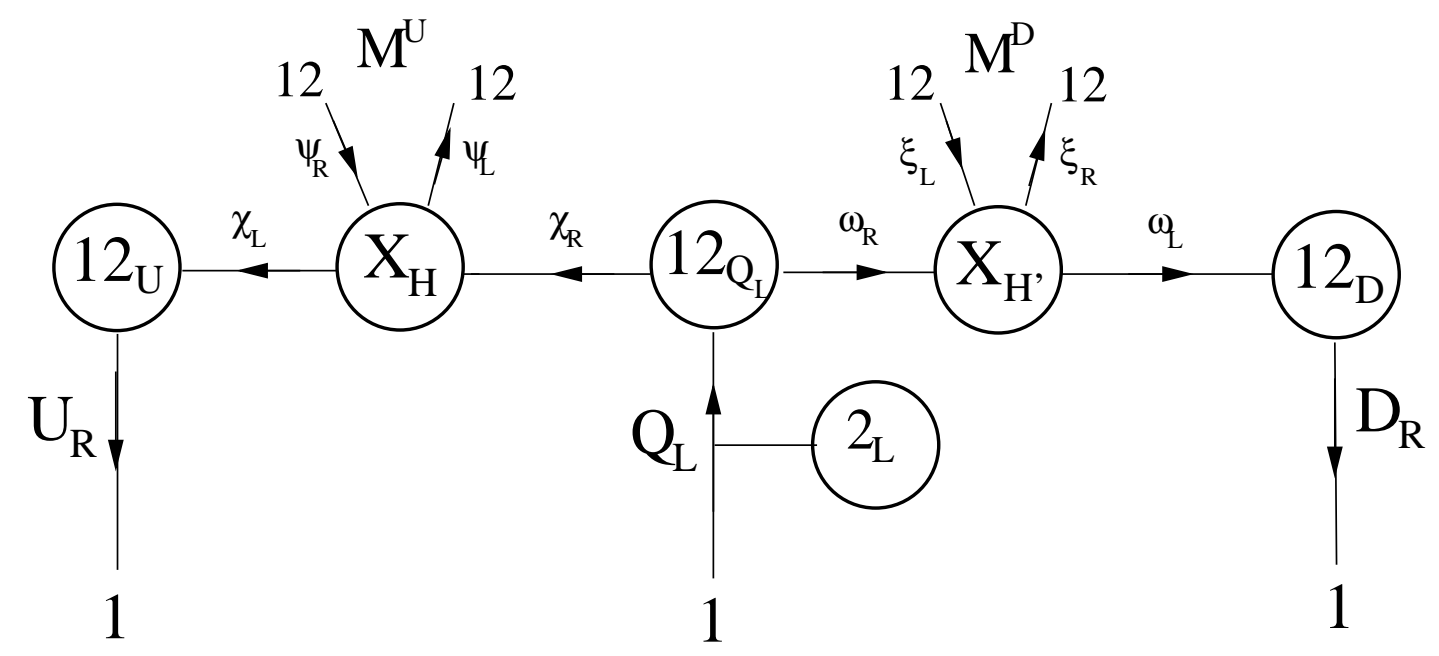

Figure 3: A flawed dynamical model of the universal see-saw resulting from broken flavour symmetry displayed in moose notation.

\section{Dynamics From Broken Flavour Symmetry}

There is an alternative possible flavour structure for the four fermion interactions driving EWS breaking that allow the leptons to condense and hence acquire masses but without giving rise to the light pNGB found in Sections 2 and 3 above. That is to allow the index $i$ on $Q_{L}, U_{R}$ and $D_{R}$ to run over the full possible set of fermion flavours. In other words, in this model the index $i$ runs over the full $S U(12)$ flavour symmetry of the SM where the 12 flavours are three families of three colours of quarks and the three families of leptons. We introduce additional $\chi^{i}\left(\omega^{i}\right)$ and $\psi^{i}\left(\xi^{i}\right)$ electroweak singlets with a similar pattern of singlet masses to those of the model of Section 2. The colour and hypercharge interactions are weakly gauged subgroups of the $S U(12)$ flavour symmetry of the SM particles and the electroweak singlet sectors. The four fermion interaction

$$
\bar{Q}_{L}^{i} \chi_{R}^{i} \bar{\chi}_{R}^{j} Q_{L}^{j}+\bar{Q}_{L}^{i} \omega_{R}^{i} \bar{\omega}_{R}^{j} Q_{L}^{j}
$$

drives electroweak symmetry breaking through the condensate $\left\langle\bar{Q}_{L}^{i}\left(\chi_{R}^{i}+\omega^{i}\right)\right\rangle$. The $S U(2) \times$ $S U(2)$ chiral symmetry of the fermion doublets is broken to the vector subgroup producing three Goldstone bosons that are all eaten by the $W$ and $Z$ bosons. In this case there are two charged Higgs bosons, two neutral Higgs and a massive pseudo-scalar, as in a standard two Higgs doublet model, with masses of order $(400-650) \mathrm{GeV}$ and no pNGBs, which makes this model phenomenologically more appealing than the one in the previous section.

It is natural to promote this idea to a moose model based on that of Section 3. One might propose the moose model of Fig. 包, a variant of the technicolour model of Ref. [11]. We 
expect again that the dynamics is such that the $S U(X)$ hypercolour groups become strong and break their chiral symmetries in the few $\mathrm{TeV}$ range. The chiral flavour groups are also assumed to be strong at this scale. Amid these dynamics the condensates $\left\langle Q_{L}^{i}\left(\chi_{R}^{i}+\omega_{R}^{i}\right)\right\rangle$, $\left\langle U_{R}^{i} \chi_{L}^{i}\right\rangle$ and $\left\langle D_{R}^{i} \omega_{L}^{i}\right\rangle$ are assumed to form. Mass matrices put in by hand for the $\psi$ and $\xi$ fermions provide the left right connecting structure necessary to produce the SM fermion masses. This is just the universal see-saw mass scheme. The SM gauge interactions result as the vector subgroup of the gauged SU(12) chiral flavour symmetries and any weakly gauged subgroup of the $\psi$ and $\xi$ fermions global $S U(12)$ symmetry groups. We do not show these weakly gauged subgroups in the figure for ease of display. The electroweak symmetry breaking VEVs do not generate any pNGBs since the 3 NGBs arising from the breaking of the $S U(2)_{L} \times S U(2)_{R}$ chiral group are eaten by the $W$ and the $Z$ gauge bosons. However, the hypercolour dynamics will generate 575 NGB, corresponding to the breaking of the $S U(24)_{L} \times S U(24)_{R}$ chiral symmetry of the $\chi$ 's and the $\psi$ 's. Of these, only 286 are eaten by the broken flavour symmetry groups. The remainder are, just as in the quark moose, potentially light although they are singlets under the SM interactions. As in that model, these light states are a result of the particular implementation of the singlet sector and not an automatic consequence of the flavour universal models. The model again has a GIM mechanism that suppresses FCNCs.

A more serious problem with the present model resides in the fact that anomaly cancellation for the gauged $S U(12)$ groups requires $X=1$. Attempts to make these groups non-abelian by the inclusion of new EWS singlet fermions transforming under the $S U(12)$ groups give rise to additional pNGBs, which may be unacceptably light (see Ref. [11 for attempts in these directions).

We present Fig 3, although it is unsatisfactory, because it helps to visualize the dynamics we envisage might be behind the model presented first at the four fermion level. A flavour universal EWS breaking scenario is possible without pNGBs and will most likely be associated with a large, broken, chiral, gauged flavour symmetry of at least the left handed SM fermions. The hypercolour dynamics is not essential to the basic idea and may at least temporarily be replaced by Higgs bosons in the absence of model building ingenuity.

\subsection{Phenomenological Considerations}

The broken flavour symmetry model is much cleaner at low energies than the model of Section 3. There are no un-eaten NGB associated with EWS breaking. The dynamics is expected to give rise to four scalar Higgs bosons and a pseudo-scalar each with masses $(400-650) \mathrm{GeV}$ which correspond to a standard two Higgs doublet model with the ratio 
of Higgs VEV, $\tan \beta=1$.

The massive flavourons are similar to those of the previous model except that they now also mediate interactions involving leptons. The GIM mechanism of the model suppresses FCNCs. One important modification with respect to the model of the previous section is the criticality condition. The couplings $\kappa_{L, R}$ obey conditions similar to Eq. (14) but with $N_{g}$ replaced by $N=12$. As a result the necessary couplings are considerably smaller than in the previous cases, which has important consequences in the phenomenology. For instance, the minimal flavouron contributions to the $\mathrm{T}$ parameter in Eq. (19) can now be smaller by roughly a factor of four, relaxing the mass bound by a factor of two. On the other hand, somewhat more stringent limits come from neutral current processes involving effective contact terms of the form $\ell \ell q \bar{q}$. We concentrate on $\ell=e$, where most of the experimental information is. The effective eeqq interactions are parametrized by the standard expression 20]

$$
\begin{aligned}
\mathcal{L}_{\mathrm{NC}}=\sum_{q} & \left\{\eta_{L L}^{e q}\left(\bar{e}_{L} \gamma_{\mu} e_{L}\right)\left(\bar{q}_{L} \gamma^{\mu} q_{L}\right)+\eta_{R R}^{e q}\left(\bar{e}_{R} \gamma_{\mu} e_{R}\right)\left(\bar{q}_{R} \gamma^{\mu} q_{R}\right)\right. \\
& \left.+\eta_{L R}^{e q}\left(\bar{e}_{L} \gamma_{\mu} e_{L}\right)\left(\bar{q}_{R} \gamma^{\mu} q_{R}\right)+\eta_{R L}^{e q}\left(\bar{e}_{R} \gamma_{\mu} e_{R}\right)\left(\bar{q}_{L} \gamma^{\mu} q_{L}\right)\right\}
\end{aligned}
$$

The exchange of the $S U(12)$ flavourons generates the first two terms, whereas the $L R$ and $R L$ terms arise only as a consequence of one loop mixing of the $L$ and $R$ gauge bosons, which is suppressed by two powers of the masses, relative to the $L L$ and $R R$ coefficients. The $L L$ and $R R$ coefficients take the form

$$
\begin{aligned}
\eta_{L L}^{e q} & =\frac{\pi \kappa_{L}}{M_{F_{L}}^{2}} . \\
\eta_{R R}^{e d} & =\frac{\pi \kappa_{R}}{M_{F_{R}}^{2}} .
\end{aligned}
$$

where $q=u, d$. The $S U(2)_{L}$ relations naturally resulting in this model imply that the $R R$ interactions of the $u$ quark involve neutrinos instead of charged leptons. This mismatch results in potentially dangerous contributions to which Atomic Parity Violation (APV) as well as neutrino scattering experiments are especially sensitive. The most constraining bounds come from the APV experiments. The resulting contribution to the atomic weak charge is given by [2]

$$
\Delta C_{q}=\frac{v^{2}}{2}\left(\eta_{R R}^{e q}-\eta_{L L}^{e q}\right)
$$

Thus, assuming that the couplings and masses of the left and right handed sector are similar, the effect largely cancels in the eedd interactions. This is not the case for eeuu. From the most recent measurements [21] we obtain the $3 \sigma$ bound

$$
\eta_{L L}^{e u}<0.22
$$


which, for the critical value of the flavouron coupling translates into $M_{F}>2 \mathrm{TeV}$. Looser bounds on $M_{F}$ are obtained from the $\nu N$ scattering experiments, as well as from HERA, LEPII and Drell-Yan processes at the Tevatron. Thus, a scale of a few TeV for the dynamics of this model is not in contradiction with experimental observations.

\section{Conclusions}

We have presented model building ideas that extend the top condensate see-saw model of Ref. [7] to include realistic mass generation for all the SM fermions. In these models all the electroweak doublets of the SM play an equal role in breaking EWS, with their mass differences resulting from different mass mixings in a heavy EWS singlet fermion sector. This effectively separates the questions of EWS breaking and the origin of fermion masses, deferring the latter to higher energy scales. As a result, the origin of the dynamics of fermion masses is removed as a source of contamination of the precision electroweak variables $S$ and $T$. The mass mixings of the EWS singlet fermions, that are the seed for the standard model fermion masses, may be generated at much higher scales than the weak scale without inducing further fine tuning in the production of the weak scale.

We presented two classes of models, based on broken gauged family symmetry in one case and broken gauged flavour symmetry in the other. In both cases the resulting composite Higgs sector is relatively light, with masses in the range $m_{h}=(400-700) \mathrm{GeV}$. In gauged family models, the breaking of the large chiral symmetries in the SM fermion sector leads to the presence of a large number of scalars and pseudo-scalars with masses in this range, resulting in constraints from the measurements of $R_{b}$ and FCNC processes. On the other hand, the scalar sector of the broken flavour symmetry models is more economical, with no pNGBs, and corresponds to the two Higgs doublet model with $\tan \beta=1$.

Both scenarios involve extensions of the gauge sector and imply the existence of massive gauge bosons associated with the breaking of either the family or flavour symmetries, the flavourons. These are strongly coupled to the SM fermions and thus have a very rich phenomenology at present and future high energy colliders. We have seen that the mass scale of the flavourons can be as low as a few $\mathrm{TeV}$ and still satisfy all existing phenomenological bounds. Therefore, the experiments to take place at the Fermilab Tevatron Collider, as well as the CERN LHC will be sensitive to a variety of signals associated with these states, as well as with the colourons already present in other models of dynamical EWS breaking. 


\section{Acknowledgments}

The authors are grateful to the Aspen Center for Physics where this work was begun and to S. Chivukula, B. Dobrescu, C. Hill and J. Terning for useful discussions. This work was supported in part by the U.S. Department of Energy under contracts \#DE-FG02-91ER40676 and \#DE-FG02-95ER40896 and the University of Wisconsin Research Committee with funds granted by the Wisconsin Alumni Research Foundation.

\section{References}

[1] S. Weinberg, Phys. Rev. D19 1277 (1979); L. Suskind, Phys. Rev. D20 2619 (1979); E. Farhi and L. Susskind, Phys. Report 74 No.3 277 (1981); S. Dimopolous and L. Susskind, Nucl. Phys. B155 237 (1979); E. Eitchen and K. Lane, Phys. Lett. B90 125 (1980).

[2] M.E. Peskin and T. Takeuchi, Phys. Rev. Lett. 65964 (1990); M.E. Peskin and T. Takeuchi, Phys. Rev. D46 381 (1992).

[3] Y. Nambu, "New Theories In Physics", Proc. XI Warsaw Symposium on Elementary Particle Physics, (ed. Z. Adjuk et al., publ. World Scientific, Singapore, 1989); V.A. Miransky, M.Tanabashi and M. Yamawaki, Phys. Lett. B221 (1989) 177; R.R. Mendel and V.A. Miransky, Phys. Lett. B 268384 (1991); W.A. Bardeen, C.T. Hill and M.Lindner, Phys. Rev. D41 1647 (1990).

[4] C. T. Hill, Phys. Lett. B345, 483 (1995).

[5] G. Buchalla, G. Burdman, C. T. Hill and D. Kominis, Phys. Rev. D53, 5186 (1996); G. Burdman and D. Kominis, Phys. Lett. B403, 101 (1997); K. Lane and E. Eichten, Phys. Lett. B352, 382 (1995); K. Lane, Phys. Rev. D54, 2204 (1996).

[6] S. Chivukula, B. Dobrescu and J. Terning, Phys. Lett. 353, 289 (1995); T.W. Appelquist, N. Evans and S.B. Selipsky, Phys. Lett. B374 145 (1996).

[7] B. Dobrescu and C. T. Hill, Phys. Rev. Lett. 812634 (1998); R.S. Chivukula, B.A. Dobrescu, H. Georgi and C.T. Hill, hep-ph/9809470.

[8] S. F. King, Phys. Rev. D45, 990 (1992).

[9] H. Georgi, "Technicolor and Families", Proc. 1990 International Workshop On Strong Coupling Gauge Theories And Beyond, (ed. T. Muta and K. Yamawaki, publ. World Scientific, Singapore,1991); H. Georgi, Nucl. Phys. B416 699 (1994). 
[10] N. J. Evans, S. F. King and D. A. Ross, Z. Phys. C60, 509 (1993).

[11] L. Randall, Nucl. Phys. B403 122 (1993).

[12] R.S. Chivukula and H. Georgi, Phys. Lett. B99 188 (1987); R.S. Chivukula, H. Georgi, L. Randall, Nucl. Phys. B292 93 (1987).

[13] M. Luty, Phys. Lett. B292 113 (1992).

[14] R.S. Chivukula, A.G. Cohen and E.H. Simmons, Phys. Lett. B380, 92 (1996); E. H. Simmons, Phys. Rev. D55, 1678 (1997) and M. B. Popovic and E. H. Simmons, Phys. Rev. D58, 095007 (1998).

[15] H. Pagels and S. Stokar, Phys. Rev. D20 2947 (1979).

[16] M.E. Peskin, Nucl. Phys. B 175197 (1980).

[17] J. Erler and P. Langacker, in "Review of Particle Physics", Eur. Phys. J.C3, 1 (1998).

[18] C. T. Hill and S. J. Parke, Phys. Rev. D49, 4454 (1994).

[19] Petr Gaidarev, for the CLEO collaboration, presented at the "Workshop on Heavy Quarks at Fixed Target, HQ98, Fermilab, October 9-12, 1998”.

[20] E. Eichten, K. Lane and M. Peskin, Phys. Rev. Lett. 50, 811 (1982).

[21] For a recent update see D. Zeppenfeld and K. Cheung, hep-ph/9810277 in "Proceedings of the WEIN Symposium, Santa Fe, NM, June 14-21, 1998", and references therein. 